\title{
TMR SELECTIVA Y RECONFIGURACIÓN PARCIAL DINÁMICA PARA MEJORAR LA TOLERANCIA A FALLOS DE CIRCUITOS EMBARCADOS
}

\author{
Federico Fernández ${ }^{1}$, Juan Carlos Fabero ${ }^{2}$ y Hortensia Mecha ${ }^{2}$ \\ ${ }^{1}$ Facultad Politécnica-UNA, San Lorenzo, Paraguay \\ ${ }^{2}$ Facultad de Informática, Univ. Complutense, Madrid, Spain
}

\begin{abstract}
RESUMEN
La evolución tecnológica de los circuitos integrados pasa por el aumento de la integración de los dispositivos en menor espacio, menor consumo de potencia y el aumento en la frecuencia de operación. Debido al aumento de estas capacidades son cada vez más numerosos los dispositivos de alta complejidad que se utilizan en los sistemas de navegación aérea, guías de misiles y aeronaves espaciales. Estos sistemas tienen la particularidad de que no admiten fallos en su funcionamiento por las consecuencias que pueden tener. Asimismo es cada vez más creciente el uso de dispositivos reconfigurables en estos sistemas por la facilidad de diseño, el tiempo reducido de pruebas y por sobre todo la posibilidad de reconfiguración dinámica. A medida que un dispositivo se aleja de la superficie terrestre existe la posibilidad que partículas de alta energía puedan impactar en estos dispositivos produciendo daños que afectarán el funcionamiento de los mismos. Estos se denominan SEE (Single Event Effects), que son el resultado del impacto de partículas energéticas en zonas sensibles de dispositivos y circuitos integrados. Para enfrentar este problema es necesaria la utilización de técnicas que mejoren la robustez de los dispositivos ante la aparición de estos efectos. Uno de ellos es el TMR (Triple Modular Redundancy) que permite la recuperación del funcionamiento de los sistemas digitales ante la aparición de los efectos del SEE pero a un alto costo en la utilización de recursos y consumo de potencia. El TMR selectivo sin embargo se aplica solo a aquellas partes que son más sensibles a los efectos del SEU.
\end{abstract}

\section{PALABRAS CLAVE}

Hardware Reconfigurable, FPGA, SEE, TMR Selectivo

\section{INTRODUCCION}

La utilización del hardware reconfigurable es cada vez más frecuente en el diseño de circuitos complejos debido a las herramientas disponibles para reducir los tiempos de diseño, prueba y depuración del circuito final. También ofrece la ventaja de que se puede modificar un diseño realizando los cambios sobre el circuito final ya que en el caso de las FPGA lo que se programa no es software sino hardware, pudiendo el circuito final ser objeto de una optimización constante a medida que pasa el tiempo. Esta capacidad de modificar un diseño dentro de una FPGA se denomina reconfiguración pudiendo ser, según el modelo, estática o dinámica. Los SEE (Single Event Effects) son efectos producidos por el impacto de las partículas de alta energía provenientes del espacio exterior. Debido al aumento en la integración de los dispositivos y a la disminución de su tensión de funcionamiento, el impacto de estas partículas puede producir desde la inducción de ruido en los componentes, el mal funcionamiento o el daño total de los mismos. Por ello se deben encontrar mecanismos que permitan a los dispositivos recuperarse en caso de sufrir algún daño debido al impacto de las partículas de alta energía. Cuando se utilizan las FPGA en los sistemas de navegación y demás circuitos auxiliares en dispositivos de vuelo y aeronaves espaciales los mismos deben tener una capacidad de recuperación ante la presencia de daños provocados por el impacto de estas partículas, que pueden provocar errores de dos tipos: soft errors, cuyos efectos no son permanentes y se denominan SEU (Single Event Upset), y los hard errors, cuyos efectos son la inutilización de la zona afectada y se denominan SEL (Single Event Latchup), debiendo en ambos casos tomar medidas correctoras para recuperar el normal funcionamiento de todo el sistema. Por ello, a fin de garantizar la robustez del sistema se buscan técnicas que garanticen una respuesta a los fallos generados por estas partículas. 
Este trabajo está organizado de la siguiente forma: En la sección II se hace una breve descripción del hardware reconfigurable. La sección III describe los diferentes efectos que pueden producir los impactos de las partículas cargadas. En la Sección IV se describe lo que es el TMR. En la sección V describimos las herramientas disponibles y en la sección VI el estado actual del proyecto.

\section{HARDWARE RECONFIGURABLE}

En esencia, la FPGA es un conjunto de recursos hardware más una memoria de configuración que determina cómo se conectan entre sí los diferentes recursos para ejecutar una tarea. Algunos modelos incorporan la posibilidad de configurar uno o más procesadores empotrados lo que aumenta aún más sus posibilidades de diseño y potencia de cálculo. Cuando se realiza un diseño digital, se utilizan los recursos hardware que la FPGA brinda para interconectar los mismos con el fin de implementar el diseño de interés. Normalmente estos diseños están formados por diferentes módulos que interactúan entre sí teniendo los mismos una funcionalidad específica. El tamaño de estos módulos estará determinado por los recursos que necesita para su función específica. Una vez sometido a pruebas y simulaciones el diseño está listo para ser sintetizado y convertido a un archivo de configuración. Este archivo recibe el nombre de bitstream y, una vez que ha sido cargado en la FPGA, el diseño está listo para su utilización. Si por algún motivo se desea modificar una tarea, simplemente se debe realizar el nuevo diseño y mediante el bitstream cargarlo nuevamente en la FPGA para su ejecución. A esto se le llama reconfiguración y es lo que hace a la FPGA muy atractiva con respecto a otras tecnologías de diseño digital. Inicialmente para la reconfiguración de la FPGA cuando se modificaba un diseño se borraba totalmente el anterior denominándose a este procedimiento reconfiguración estática. Posteriormente aparecieron nuevos modelos que permiten modificar una parte determinada de la configuración sin la necesidad de borrar todo el diseño original, permitiendo modificar solamente aquellos módulos de interés sin detener el funcionamiento del sistema. A este procedimiento se le denomina reconfiguración dinámica. En este tipo de reconfiguración se presentan problemas asociados como la fragmentación y el retardo que esto produce en el tiempo de finalización en la ejecución de un diseño que tiene numerosas tareas que se van cargando y liberando de la FPGA. Estos problemas van aumentando a medida que el diseño crece en complejidad (Xilinx., 2004), (Blodget, B. et al., 2004).

\section{EFECTOS ASOCIADOS A LOS IMPACTOS DE LAS PARTICULAS DE ALTA ENERGÍA}

La evolución tecnológica pasa por el aumento de la integración de los dispositivos, menor consumo de potencia y el aumento en la frecuencia de operación de los mismos. Esto se traduce en un aumento potencial de la vulnerabilidad a los efectos de radiaciones debido al impacto de las partículas cargadas de alta energía. En ciertas aplicaciones en donde no se admiten fallos, este factor se debe tener muy en cuenta. Aquí aparecen los efectos que estos impactos producen, como los SEE (Single Event Effects), que son el resultado del impacto de partículas energéticas en zonas sensibles de dispositivos y circuitos integrados. Estos impactos actúan creando una carga libre en el silicio, que puede resultar en un pulso de corriente de corta duración pero intensa, con consecuencias tales como la generación de ruido en las señales, cambio en el contenido de celdas de memorias y la creación de cortocircuitos destructivos, siendo los efectos más notorios si el impacto se produce cerca de un área sensible. Los SEE tienen una clasificación que depende de los efectos que los mismos producen.

Single Event Upset (SEU): Se produce cuando por efectos del impacto de la partícula cargada se produce el cambio del valor de un bit.

Múltiple Bit Upset (MBU): Sus efectos son mayores que el anterior porque son varios los bits que cambian de valor dentro del circuito. 
Single Event Transient (SET): Produce un pulso de corta duración y afecta a circuitos combinatorios, aunque dicho pulso puede ser capturado por un circuito secuencial si se produce a la entrada de éste y coincide con un pulso de reloj.

Single Event Latch-Up (SEL): Este tipo de efectos produce la activación de señales parásitas dentro de los circuitos. Puede ser destructivo.

Funtional Interruption (SEFI): Produce la pérdida de la funcionalidad por lo cual se precisa del reset del sistema (Velazco, R., 2013).

Los SEE son asociados a las misiones espaciales, debido a la ausencia del escudo atmosférico que envuelve el planeta y que protege a la superficie de las incidencias de estas partículas de alta energía. Se espera que los efectos de los SEE se hagan sentir más en el futuro debido a que la sensibilidad de circuitos integrados puede aumentar debido a diferentes parámetros. Uno de ellos es la tensión de trabajo de estos circuitos, ya que al ser cada vez más pequeños, el impacto de una partícula cargada puede generar un pico de voltaje que puede inducir ruido dentro del circuito cambiando los valores digitales de los mismos. Este impacto puede producir cambio de uno o varios bits simultáneamente. Otro aspecto a tener en cuenta es la frecuencia de funcionamiento, ya que cuando más elevadas son, un impacto afectará a más bits, con más razón si el dispositivo está en estado de transmisión o recepción. Finalmente está la altura a la que se encuentra el dispositivo, ya que a medida que ésta aumenta también lo hace la posibilidad de que ocurra algún evento de este tipo. También hay que mencionar que todos los bloques combinatorios, memorias y procesadores que están presentes en los circuitos de alta complejidad que componen los sistemas de aviación, misiles y navegación espacial pueden sufrir cualquiera de las formas del SEE.

Por ello a la hora del diseño de estos dispositivos complejos se deben considerar los mecanismos que pueden mitigar o anular los efectos del SEE entre las que se pueden distinguir.

- Triple Modular Redundancy (TMR) Este esquema de mitigación utiliza tres circuitos lógicos idénticos que realizan la misma tarea en paralelo con las salidas correspondientes que se comparan a través de un circuito de votación mayoritario.

- Time Redundancy. Se utiliza cuando se diseñan sistemas tolerantes a fallas que requerirían gran cantidad de hardware adicional utilizándose, en cambio, más tiempo en el cálculo de los mismos. Se utiliza en sistemas en las cuales el tiempo es menos importante que el uso del hardware y se utiliza para distinguir entre fallas transitorias o permanentes.

- Software Redundancy se utiliza en sistemas donde los módulos de programación considerados críticos se duplican o triplican para asegurar la detección de una falla.

Existes otras técnicas para mitigar los efectos del SEE todas ellas son complementarias. Nuestro trabajo estará basado en el TMR. (Berrojo, L. et al., 2002), (Criswell, T. et al., 1984), (Dixit, A. and Wood, A., 2011).

\section{QUÉ ES EL TMR TRIPLE MODULAR REDUNDANCY}

Todos los sistemas digitales complejos necesitan algún mecanismo de protección ante la presencia de impactos de partículas de alta energía que puede alterar su configuración y, por tanto, su buen funcionamiento. Una vez que estos impactos se produzcan sobre estos sistemas se debe activar un mecanismo que favorezca la tolerancia y mitigación de esos efectos. El TMR (Triple Modular Redundancy) es uno de los mecanismos más utilizados en la actualidad. Este esquema de mitigación utiliza tres circuitos lógicos idénticos que realizan la misma tarea en paralelo con las salidas correspondientes que se comparan a través de un circuito de votación mayoritario. El ejemplo más común de TMR es un circuito C que se ha triplicado y que tiene un votador agregado en su salida, Figura 1. Como en los circuitos complejos el hecho el triplicar 
el número de los mismos producen dos consecuencias. Por un lado al aumentar el número de recursos que se utilizan en el diseño también aumenta el consumo de energía, pero protegiendo efectivamente los módulos del circuito que utilizan estos elementos. Y por el otro no se protege a la lógica combinatoria que conecta los circuitos en el diseño.

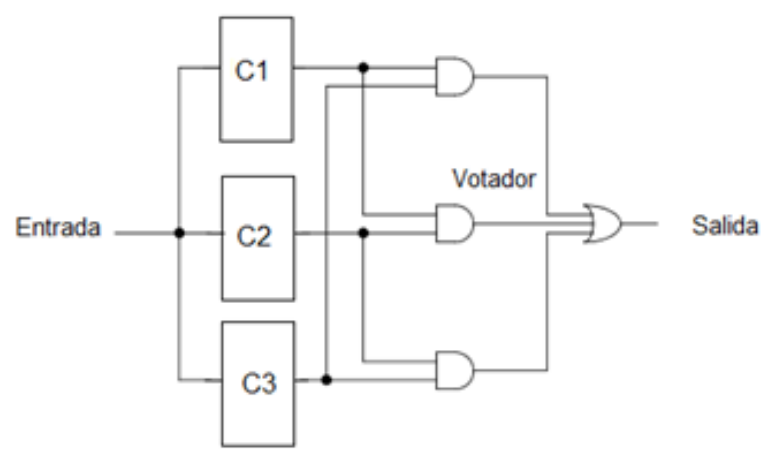

Figura 1. Triplicación de circuitos utilizando TMR

Los efectos de los SEE no se limitan a los registros en diseños digitales, sino que también están presentes en la lógica combinatoria para la cual hay varios esquemas de protección a ser considerados. Estos esquemas tratan sobre todo problemas técnicos transitorios en la lógica combinatoria que podrían provocar trastornos en los elementos secuenciales. Adicionalmente, las FPGA también son susceptibles a los efectos de los SEE en la memoria de configuración SRAM, siendo este efecto más severo porque puede afectar al propio diseño. Por ello para elevar la eficiencia de un sistema de protección se debe tener en cuenta la lógica completa del diseño, tanto la parte secuencial como la combinatoria.

Otra forma de mitigar los efectos de las partículas cargadas en los sistemas digitales es mediante la replicación a nivel modular y teniendo a la salida un sistema de votación que permita determinar si ha habido un error en el funcionamiento de alguno de estos módulos. En este caso un módulo puede representar el diseño completo para un dispositivo en particular o un subcomponente de ese diseño. Este es un medio muy efectivo de mitigación SEE que es fácil de implementar y se puede realizar por completo en un solo dispositivo, siempre que los módulos no utilicen más de un tercio del dispositivo total, Figura 2.

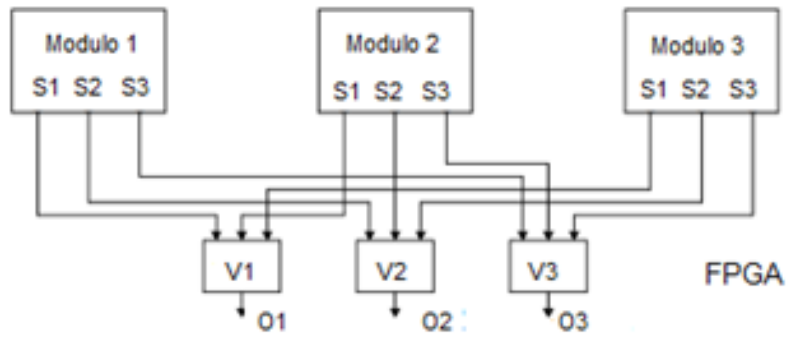

Figure 2. Triplicación de módulos utilizando TMR

Otra alternativa es la redundancia triple de dispositivos. Tiene la más alta confiabilidad para detectar diferentes fallas debidos a los efectos del SEE incluyendo fallas de los mismos dispositivos. Sin embargo, ésta es también la solución más costosa, Figura 3. Una forma de disminuir el costo en recursos y el consumo de potencia en los dispositivos que son triplicados es que si los mismos son de gran complejidad y tamaño ver la posibilidad de determinar qué partes de dichos diseños son más susceptibles de ser afectados por el impacto de las partículas. Hay que tener presente que también los propios fabricantes de FPGA ofrecen soluciones para mitigar los efectos del SEE que pueden presentarse durante su funcionamiento, (Habinc, S., 2002), (Mahatme, N. et al., 2011), (Morgan, K. et al., 2005). 


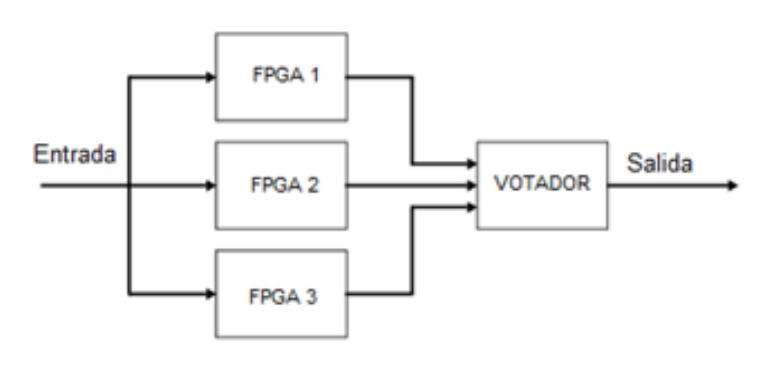

Figura 3. Triplicación de dispositivos

\section{HERRAMIENTAS PARA TMR}

Existen dos maneras de mejorar la robustez en el funcionamiento de un circuito. El primero de ellos es utilizando las herramientas que suministran los propios fabricantes y la otra diseñándolo a medida. En el primero como nuestro proyecto gira en torno a un modelo de la serie 7 de Xilinx las herramientas deben estar basadas en el entorno de desarrollo Vivado. MicroBlaze TMR Subsystem v1.0 proporciona herramientas de recuperación basado en MicroBlaze, permitiendo el funcionamiento del sistema aun después de encontrar un error, asegurando la fiabilidad del sistema. Diseñar este tipo de herramientas es muy complejo en términos de tiempo y número de ensayos, si se va recurrir al método tradicional de programación utilizando lenguajes como Verilog o VHDL. Pero mediante el uso de módulos de propiedad intelectual IP de Vivado se simplifica la creación de los módulos TMR, ya que la creación de Microblaze y las herramientas de desarrollo TMR se realizan llamando a estas herramientas en el entorno de desarrollo Vivado. Para comprobar la eficacia de las técnicas TMR se pueden realizar simulaciones mediante inyección de errores, bien con las herramientas proporcionadas por los fabricantes (Xilinx, 2017), (Xilinx, 2017) o mediante software específico, (Claramunt, J. A., 2016), (Serrano, F. et al., 2009), (Rodríguez Bodas, A., 2015), (Zuzarte, M. A., 2014). Hemos elegido para el desarrollo del proyecto la placa Nexys 4 que tiene empotrado una FPGA Artix 7 que además de tener numerosas posibilidades para el desarrollo de circuitos combinacionales complejos, nos permite el diseño de procesadores empotrados como MicroBlaze y por sobre todo realizar reconfiguración parcial dinámica.

\section{ESTADO ACTUAL DEL PROYECTO}

Los pasos previos del proyecto han sido el desarrollo de circuitos con reconfiguración parcial dinámica obteniéndose buenos resultados. El siguiente paso es realizar un banco de filtros FIR de 32 bits en la que pretende implementar un banco de filtros reconfigurables a fin de utilizarlo como diseño de pruebas. Posteriormente iremos implementando los objetivos específicos del proyecto como ser implementación y extracción de resultados experimentales de Técnicas de protección TMR selectivas, la realización de una propuesta de ampliación y mejora de las técnicas TMR tradicionales, el estudio e implementación de técnicas de planificación y ubicación dinámica de tareas sobre plataformas reconfigurables, la extracción de resultados experimentales en cuanto a la mejora de la habilidad y los tiempo de ejecución para las técnicas propuestas y finalmente la presentación de una propuesta de mejoras arquitectónicas en las plataformas reconfigurables.

\section{AGRADECIMIENTO}

Este trabajo fue realizado con financiamiento de los fondos del Programa PROCIENCIA del CONACYT PINV15-99 y de la F.P. - U.N.A. 


\section{REFERENCIAS}

Berrojo, L. Corno, F. Entrena, L. Gonzalez, I. López, C. Sonza Reorda, M. and Squillero, G. 2002 An industrial environment for high-level fault tolerant structures insertion and validation. In Proc. 20th IEEE VLSI Test Symp., pages $229-236$.

Blodget, B. Bobda, C. Huebner, M. and Niyonkuru, 2004. A. Partial and Dynamically Reconfiguration of Xilinx Virtex- II FPGAs citeseerx.ist.psu.edu/showciting?cid=4356857.

Claramunt Pérez, J. A. 2016. Nessy 7.0: entorno de inyección de fallos para Artix-7. https://eprints.ucm.es/38458/.

Criswell, T. Measel, P. and Wahlin, K. L. 1984. Single event upset testing with relativistic heavy ions. IEEE Trans. Nucl. Sci., NS-31(6):1559 1561, December 1984.

Dixit, A and Wood, A. 2011.The impact of new technology on soft error rates. In IEEE Int. Reliability Physics Symp., pages 5B.4.15B.4.7., April 2011.

Habinc, S. 2002. Functional Triple Modular Redundancy (FTMR). http://microelectronics.esa.int/techno/fpga_003_01-02.pdf.

Mahatme, N. Jagannathan, S. Loveless, T. Massengill, L. Bhuva, B. Wen, S. and Wong R. Comparison of combinational and sequential error rates for a deep submicron process. IEEE Trans. Nucl. Sci., 58(6):2719 2725, December 2011.

Morgan, K. Carey, M. Graham, P. Johnson, E. Pratt, B. and Wirthlin, M. SEU-induced persistent error propagation in FPGAs. IEEE Trans. Nucl. Sci., 52(6):2438 2445, December 2005.

Rodríguez Bodas, A. 2015. Sistema de inyeccion de fallos para evaluación de la fiabilidad en FPGAS https://core.ac.uk/download/pdf/79176762.pdf.

Serrano, F. Alaminos, V. Clemente, J.A. Mecha H. y Liu, S.F. 2009. NESSY: Una plataforma de inyeccion de errores para una FPGA Virtex-5. http://www.jornadassarteco.org/js2012/papers/paper_56.pdf.

Velazco, R., 2013. https://web.fdi.ucm.es/posgrado/conferencias/RaoulVelazco2013-slides.pdf.

Xilinx Inc. MicroBlaze Triple Modular Redundancy (TMR) Subsystem v1.0. Vivado Design Suite PG268 October 4, 2017. Xilinx.

Xilinx Inc. TMRTool User Guide TMRTool Software Version 13.2UG156 (v3.1.2) June 23, 2017. Xilinx.

Xilinx Inc. Two flows for partial reconfiguration: Module Based and difference based, 2004. Application Note 290. http://www.xilinx.com.

Zuzarte, M. A. 2014. A tool for run time soft error fault injection into FPGA circuits https://pdfs.semanticscholar.org/1e98/2091eb77da7c7006cdea93741187fd62fcb2.pdf. 